\title{
1/f Spin Noise and a Single Spin Detection with STM
}

\author{
Yishay Manassen ${ }^{1}$ and A. V. Balatsky² \\ 1 Department of Physics and the Ilse Katz Center for Nanometer Scale Science and Technology, \\ Ben Gurion University, Beer Sheva, 84105, Israel; ${ }^{2}$ Theoretical Division, \\ Los Alamos National Laboratory, Los Alamos, New Mexico 87545 USA
}

(Dated: April 22, 2003)

\begin{abstract}
We propose a novel mechanism for single spin detection based on the $1 / \mathrm{f}$ spin current noise. We postulate the $1 / \mathrm{f}$ spin noise for the tunneling current, similar to the ubiquitous 1 /f noise in magnetic systems. Magnetic coupling between tunneling electrons and localized spin $\mathbf{S}$ then leads to the peak at Larmor frequency in the power spectrum of the electric current fluctuations $I_{\omega}^{2}$. The elevated noise in the current spectrum will be spatially localized near the magnetic site. The difference in the power spectra taken at the Larmor frequency and elsewhere would reveal the peak in the spectrum. We argue that signal to noise ratio for this mechanism is on the order one.

In addition we discuss the asymmetric lineshapes observed regularly with this measurement. We show that such lineshapes are in accordance to the random sampling done with the tunneling electrons. Yet, this predicts a linewidth at least one order of magnitude larger than observed experimentally which is likely to be due to electrostatic repulsion between the tunneling electrons and temporal correlations in the tunneling process.

PACS numbers: $76.30 .-\mathrm{v}, 07.79 . \mathrm{Cz}, 75.75 .+\mathrm{a}$
\end{abstract}

\section{1/F SPIN NOISE}

The phenomenon of 1 /f (flicker) noise is known for almost 80 years [1]. It describes the deviation from the flat spectral density expected from a current made of uncorrelated charge carriers - at low frequencies. In this range, the spectral density was found to obey a power law of the form $1 / f^{\alpha}$ where $f$ is the frequency, and $\alpha=0.5-1.5$. Flicker noise appears in endless number of electronic devices, in music [2] in ocean streams [3, 4] and in many entirely different systems. This is one of the most universal phenomena, yet, one of the largest enigmas in physical sciences.

An early explanation to this phenomenon was that the $1 / \mathrm{f}$ noise can arise from a superposition of relaxation processes [5]. In this model the noise is described as a superposition of consecutive random events, each starts at a certain time $t_{0}$ and follow a simple exponential relaxation law: $N\left(t-t_{0}\right)=N_{0} e^{-\left(t-t_{0}\right) / \tau}$. The power spectrum of one such event is a Lorentzian. The power spectrum of a large number of such consecutive random events, all with the same $\tau$ is also a Lorentzian. If, on the other hand, there is a distribution of relaxation times $P(\tau) \sim 1 / \tau$, from $\tau_{1}$ to $\tau_{2}$ than the overall spectral density will obey a power law $\sim 1 / f$ in the range between $\tau_{1}^{-1}$ and $\tau_{2}^{-1}$. A common denominator to many mechanisms proposed for different $1 / \mathrm{f}$ phenomena [6, 7] is a distribution of relaxation times.

$1 / f$ noise is ubiquitous in STM tunneling current although its origins are a source of continuous mystery that is not fully understood until now [8, 9, 10, 11]. Unlike the well known shot noise $1 / f$ noise is proportional to the square of the current such that: $\left\langle I^{2}(\omega)\right\rangle / I^{2}=$ const. Where $\left\langle I^{2}(\omega)\right\rangle$ is the spectral density of current fluctuations (in units of $\mathrm{A}^{2} / \mathrm{Hz}$ ) and I is the current. Empirically, the current fluctuations in $1 / \mathrm{f}$ noise are known to obey the Hooge formula 12] $\left\langle I^{2}(\omega)\right\rangle / I^{2}=a 1 /[f N]$ where $\mathrm{N}$ are the number of current carriers in the sample and $a$ is of the order of 0.01 . The appearance of $1 / f$ noise in the STM is a surprising observation because the normal "explanation" of this noise is a fluctuating defect with a wide distribution of relaxation times. Such explanations are not suitable here, because of the extremely local nature of the measurement. The STM measurements of $1 / f$ noise (in a voltage of $-0.5 \mathrm{~V}$ ) gives a peak at zero frequency where the intensity of the current noise $\left\langle I^{2}(\omega)\right\rangle$ is 20 times larger than the Thermal (Nyquist) noise which will give the order of magnitude of the noise in higher frequencies (at room temperature) and amounts to $41 \mathrm{f} A / \sqrt{H z}$. The width of the observed $1 / f$ noise peak at zero frequency is of the order of $10-100 \mathrm{KHz}$.

A special case of $1 / \mathrm{f}$ noise relevant to the present paper is the magnetic flicker noise. In this case the noise is due to fluctuations in the magnetization. Normally, it is assumed that these fluctuations constitute an additional noise source in any magnetic system. However, low frequency magnetic noise is hard to observe experimentally and sensitive detection techniques and special systems are required. Such fluctuations were detected for the first time in spin glasses with a SQUID magnetometer [13, 14]. They were detected with SQUID also in antiferromagnetic thin films [15] and on superparamagnetic nanoparticles [16]. Other ways to detect $1 / \mathrm{f}$ magnetic noise is by following the resistance fluctuations close to a certain ferromagnetic transition (colosal magnetoresistance) [17], and to observe the electrical noise generated in small Hall probe contacts [18]. The $1 / \mathrm{f}$ noise generated in the Hall contacts is much larger than the usual 1/f noise measured in the same system which is unrelated to magnetic fluctuations. Despite the 
failure to measure spatial correlations it is obvious [18] that there is a certain coherence length such that two noise measurements done at a distance smaller than this length will give the same results.

Although we have no direct experimental evidence we claim that since conduction electrons in metal constitute also a (special) magnetic system, there should be $1 / \mathrm{f}$ magnetic fluctuations also in the spins in such a system which is also paramagnetic. ESR of conduction electrons is known for many years [19, 20. These spectra are known for their special lineshape 21] and for the narrow lines $\left(T_{1}\right.$ and $T_{2}$ are equal and large). We would like to emphasize that the sequence of individual dephasing events which are responsible for $T_{2}$ relaxation of conduction electrons are very similar to the sequence of relaxation events which are responsible for $1 / \mathrm{f}$ noise in general. Therefore, there are distributions of relaxation times that are expected to give $1 / \mathrm{f}$ magnetic fluctuations in conduction electrons in metals.

Such $1 / \mathrm{f}$ magnetic noise is expected to be of general significance in spintronics applications. We discuss here the implications of such $1 / \mathrm{f}$ magnetic noise (either in the spins of the conduction electrons or in a regular paramagnetic system) on single precessing spin detection with STM (ESR-STM). It will be shown below that this $1 / \mathrm{f}$ spin noise explains the observation of the signal from non magnetic tips and elucidates several aspects in this technique. We find that the interaction of the tunneling electrons spin with the local impurity spins simply couples the 1 /f magnetic noise with the noise of the local spin $\mathbf{S}$ thus creating a peak in the current noise at Larmor frequency.

ESR-STM is a technique that is using the extremely local nature of the STM measurement to detect the precession of isolated spin centers on the surface. When a tip of an STM is located above a paramagnetic spin center (in the presence of an external magnetic field) the tunneling current is modulated by the precession. It was shown 22, 23. that the $\mathrm{AC}$ current at the Larmor frequency is spatially localized within $0.5-1 \mathrm{~nm}$. It is the spatial localization that indicates (though it must be proved) that this technique is capable of detecting a single spin. In addition it was proved that the frequency of the signal is dependent on real time on the size of the magnetic field 24, 25]. More recently similar experiments have been done on the paramagnetic BDPA molecule 26. . A recent paper shows that ESR-STM can be done also on a TEMPO molecule, revealing the hyperfine spectrum [39]. The interest in this technique has risen sharply recently, due to the possibility to manipulate and detect a single spin [27, 33] and due to the possibility to use it for quantum computation [27, 28]. There have been many proposals for the mechanism of this phenomenon 29, 30, 31, 32, 33, 34, 35, 36. However, an experimental verification for any of the proposals is still required.

In our previous papers [30, 31] we discussed the following question: What is the role of the Heisenberg exchange interaction in ESR-STM? Under which circumstances can a tip emitting tunneling electrons with a random spin orientation create an elevated noise level at the Larmor freuqency through interaction with the single precessing spin?

It was argued in [30, 31 that existence of long time correlations in the temporal spin polarization of tunneling current is sufficient to provide the elevated noise at the Larmor frequency in the current noise. Here we further build upon this idea and show that the $1 / f$ noise in the tunneling spin current is sufficient to produce the effect. We stress that no correlations in the spin polarization of the tunneling electrons within the precession period is required to produce the elevated noise at Larmor frequency in this approach.

We discussed the spin dependent tunneling matrix element:

$$
G=G_{0} \exp \left[-\left[(F-J \mathbf{S}(t) \cdot \mathbf{s}(t)) / F_{0}\right]\right]
$$

$s$ is a spin matrix with implicit spin indices that are given by conduction electron spin operator $s^{i}=1 / 2 \sigma_{\alpha, \beta}^{i}, i=x, y, z$ , $\mathbf{S}(t)$ is the local impurity spin. In an external magnetic field it will have a random dynamics with remnant of the precession at the Larmor frequency. $F$ is the barrier height (typically $4 \mathrm{eV}$ ), $J$ is the exchange coupling and $F_{0}$ is the energy related to the distance $d$ between the tip and the surface $F_{0}=d^{2} / 8 m d^{2}$. In this term, the tunneling probability depends on the relative orientation between $\mathbf{S}$ and $\mathbf{s}$. We write:

$$
G=G_{0} \exp \left[-\left[\left(F / F_{0}\right]\right]\left(\operatorname { c o s h } \left[\left(J S / 2 F_{0}\right)+\mathbf{s}(t) \cdot \mathbf{n}(t) \sinh \left(J S / 2 F_{0}\right) .\right.\right.\right.
$$

Where $\mathbf{n}$ is a unit vector in the direction of $\mathbf{S}$.

The Hamiltonian we consider describes a spin dependent tunnneling matrix element between the tip (L electrode) and the surface ( $\mathrm{R}$ electrode)

$$
H=\sum_{\mathbf{k}, \alpha} \epsilon(\mathbf{k}) c_{L \alpha}^{\dagger}(\mathbf{k}) c_{L \alpha}(\mathbf{k})+(L \rightarrow R)+\sum_{\mathbf{k}, \mathbf{k}^{\prime}} c_{L \alpha}^{\dagger}(\mathbf{k}) G_{\alpha \beta} c_{R \beta}\left(\mathbf{k}^{\prime}\right)
$$

We assume that the magnetic field is along $\mathrm{z}$ axis: $B \| z$ with corresponding Larmor frequency $\omega_{L}=g \mu_{B} B$. The tunneling current operator will contain the spin independent part that we omit hereafter and the spin dependent part:

$$
\delta \hat{I}(t)=G_{1} \mathbf{n}(t) \mathbf{I}_{s}(t)
$$




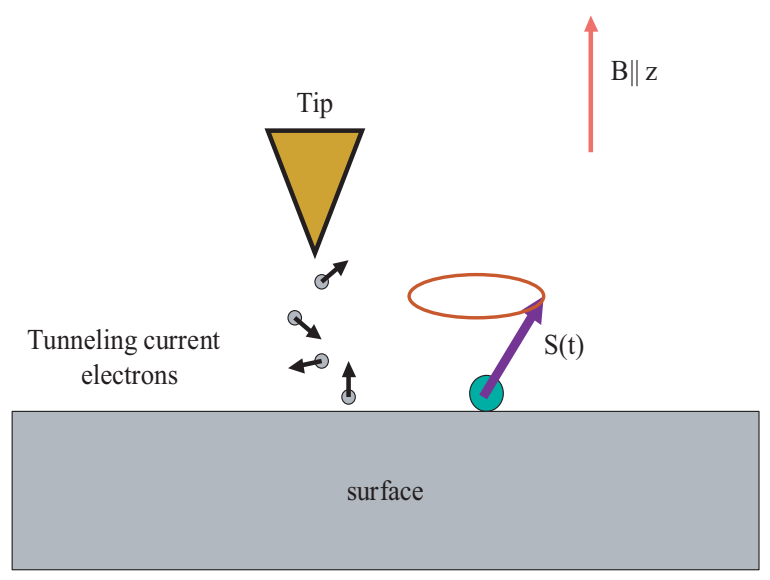

FIG. 1: Schematics of the ESR-STM experiment is shown. The fluctuations in the spin polarization of the tunneling electrons at the time scale of the precession $T$ will be nonzero and will scale as $\frac{1}{\bar{N}}$. Once the tip is positioned close to the localized spin, the exchange interaction between the localized spin and the tunneling electrons will modulate the tunneling current. The conditions in which this random modulation of the spin tunneling current with $1 / f$ spectrum will create a $\omega_{L}$ peak are discussed in the text.

where $G_{1}=G_{0} \exp \left[-\left[\left(F / F_{0}\right]\right] \sinh \left[\frac{J S}{2 F_{0}}\right]\right.$, and $\mathbf{I}_{s}(t)$ is a spin current between tip and substrate. The dc current at a given bias $V$ is : $I_{0}=g_{0} V, g_{0}=G_{0} \exp \left[-\left[\left(F / F_{0}\right]\right] \cosh \left[J S / 2 F_{0}\right]\right.$. The current-current correlator, normalized to dc current is then:

$$
\begin{aligned}
& \frac{\overline{\left\langle\hat{I}(t) \delta \hat{I}\left(t^{\prime}\right)\right\rangle}}{I_{0}^{2}}=\left(\sinh \left[\frac{J S}{2 F_{0}}\right]\right)^{2} \\
& \sum_{i, j=x, y, z}\left\langle n^{i}(t) n^{j}\left(t^{\prime}\right)\right\rangle \overline{I_{s}^{i}(t) I_{s}^{j}\left(t^{\prime}\right)}
\end{aligned}
$$

More rigorous treatment, similar to the one done in [37], where one takes into account the effect of the local spin on the mass current in the ignored terms will lead to the contribution of the same order as the term we are focusing on. Hence we use a simplified formula for the current that gives the right order of magnitude estimate.

The change in the tunneling conductance due to exchange interaction between the tunneling electrons and the localized spin leads to $\mathrm{Eq}(4): \delta I(t) \sim \mathbf{I}_{\mathbf{s}}(\mathbf{t}) \cdot \mathbf{S}(\mathbf{t})$. Only the transverse component contribute to this term $\left(S_{x}(t)\right.$ and $\left.S_{y}(t)\right)$. When the spins of the tunneling electrons are completely uncorrelated we can say that the dispersion of the tunneling current which is dependent on the spin (over one precession period T) is: $\sum_{i}\left\langle\delta I^{2}\right\rangle \sim \sum_{i=1}^{N}\left[s_{x}\left(t_{i}\right) I_{s, x}\left(t_{i}\right)\right]^{2}+$ $(x \rightarrow y) \sim<N>$. Or, the relative dispersion compared with the tunneling current will be: $\left\langle I^{2}\right\rangle / I^{2} \sim 1 /\langle N\rangle$. To estimate the magnitude of the spin dependent dispersion, namely the size of the noise which is due to the interaction with the precessing spin, we use:

$$
\left(<I^{2}>\right)^{1 / 2} / I_{0} \sim 2 / N \sinh \left[\left(J S / 2 F_{0}\right) .\right.
$$

If we take a typical value of $J=0.1 \mathrm{eV}, F=4 \mathrm{eV}, F_{0}=0.1 \mathrm{eV}$ (for $d=4 \AA$ ) and $S=1 / 2$, we get a value of 0.01 , namely rf intensity of 10 picoampere, which is within the right order of magnitude.

The spectrum is detected at the frequency domain, and by looking at the Fourier transform of the current fluctuations. This means that the spectrum is a convolution of the power spectrum of the single spin fluctuations: $<S_{i}^{2}(\omega)>=\frac{\gamma}{\left[\left(\omega-\omega_{L}\right)^{2}+\gamma^{2}\right]}$ and the power spectrum of the spin tunneling current: $\left\langle I_{s, i}(\omega) I_{s, j}(-\omega)\right\rangle=\delta_{i j}\left\langle I_{s}^{2}(\omega)>\right.$. Here, to be specific, we assumed the spectrum of a localized spin to be Lorenzian, however the argument is applicable to the case of general spectrum $<S_{i}^{2}(\omega)>$.

$$
\begin{array}{r}
\frac{\overline{\left\langle I_{\omega}^{2}\right\rangle}}{I_{0}^{2}}=\left(\sinh \left[\frac{J S}{2 F_{0}}\right]\right)^{2} \\
\sum_{i=x, y, z} \int \frac{d \omega_{1}}{2 \pi}\left\langle\left(I_{s}^{i}\right)_{\omega-\omega_{1}}^{2}\right\rangle\left\langle\left(S^{i}\right)_{\omega_{1}}^{2}\right\rangle
\end{array}
$$

If the tunneling electron spins are completely uncorrelated, then the power spectrum is white noise, and the expected signals will be scattered over the whole frequency range. In order to get a peak at the Larmor frequency, some 
correlation at zero frequency is required, namely the tunneling electron spins have to have a some temporal spin polarization in the long time limit 35].

To give an example we consider the case where several magnetic atoms are adsorbed on the tip. Then the spins of the tunneling electrons will be under the influence of slowly changing spins $\mathbf{h}_{i}$ of magnetic atoms due to exchange interaction $H_{\text {int }}=\sum_{i} J_{i} \mathbf{h}_{i}(t) \boldsymbol{\sigma}(t)=\mathbf{H}(t) \boldsymbol{\sigma}(t)$ where $J_{i}$ are the exchange couplings to the i-th atom and we define $\mathbf{H}$ as a sum of random fluctuating magnetic moments $\mathbf{h}_{i}$ of individual magnetic impurities adsorbed on the tip. Here we assume that only the "field" value $\mathbf{H}$ at the end of the tip matters as the tunneling electrons are affected by this field the most before tunneling out of the tip. This fluctuating exchange field will spin polarize the tunneling current. The correlator is $\left.<\mathbf{H}(t) \mathbf{H}\left(t^{\prime}\right)\right\rangle=\Sigma_{i}<\mathbf{h}_{i}(t) \mathbf{h}_{i}\left(t^{\prime}\right)>$. The correlation function from each impurity assuming they are independent, obeys $<\mathbf{h}_{i}(t) \mathbf{h}_{i}\left(t^{\prime}\right)>=e^{-\left(t-t^{\prime}\right) / \tau_{i}}$. Average over distribution times $\tau_{i}$ will with probability distribution $P(\tau) \sim 1 / \tau$ between $\tau_{1}$ and $\tau_{2}$, will yield the $1 /$ f noise for the field fluctuation power spectrum. This follows directly from evaluating the Lorenzian in frequency space $\frac{\tau}{\omega^{2} \tau^{2}+1}$, averaged over distribution of relaxation time $\tau$ with $P(\tau)$; e.g see Ref[7]:

$$
\left\langle\mathbf{H}^{2}(\omega)\right\rangle=\int_{\tau_{1}}^{\tau_{2}} d \tau \exp \left(i \omega\left(t-t^{\prime}\right)\right) P(\tau)\left\langle\mathbf{h}_{i}(t) \mathbf{h}_{i}\left(t^{\prime}\right)\right\rangle \sim \frac{1}{\omega}
$$

Hence the tunneling current will acquire spin polarization fluctuations. It is natural to assume that the spin fluctuations of the tunneling electrons will be proportional to fluctuating exchange field $\mathbf{H}$. The degree of spin polarization of the tunneling electron is proportional to the Zeeman energy $\left(g \mu_{B} \mathbf{H}\right) / W$ related to the total bandwidth $W \sim 1 / N_{0}$. We get

$$
\mathbf{I}_{s}(t)=\operatorname{Ig} \mu_{B} \mathbf{H}(t) N_{0}
$$

here we assumed that the effect is proportional to the tunneling current $I$. Indeed tunneling electrons will sample the random field $\mathbf{H}$ at a rate at which they tunnel and this rate is given by the electric current $I$.

We now consider the specific case when spin tunneling current $\mathbf{I}_{\mathbf{s}}$ has $1 / f$ noise component.

$$
\left\langle I_{s}^{2}(\omega)\right\rangle=\frac{C}{|\omega|}, C=a I^{2}
$$

for all components $i=x, y, z$. The magnitude of the $1 / f$ noise is to be given by $I^{2}$ up to an unknown numerical factor $a$ [12], where $N$ is absorbed in $a$ now. $1 / f$ noise is peaked at zero frequency and will provide a peak at the Larmor frequency in the convolved spectrum, Eq.(7). We also remark here that in a similar fashion one can get the $1 / f$ noise in the spin current due to $1 / f$ noise in the electronic current in a presence of a constant polarizing field. We will not consider this possibility here.

Spin tunneling current will have white noise asymptotic at high frequencies. At lower frequencies, similar to the unpolarized electric current [8, 9, 10, 11], the relaxation processes that control spin relaxation in the current will contribute. To give a physically plausible argument about the origin of the $1 / f$ noise consider relaxation time of the tunneling spin current:

$$
\left\langle I_{s, i}(t) I_{s, j}\left(t^{\prime}\right)\right\rangle=\delta_{i j} \exp \left(-\left|t-t^{\prime}\right| / \tau\right)
$$

this correlation function leads to the Lorenzian for the noise spectrum with the width $\gamma=1 / \tau:\left\langle I_{s, x}^{2}(\omega)\right\rangle=\frac{\gamma}{\pi\left(\omega^{2}+\gamma^{2}\right)}$. Now we assume that there is a distribution of relaxation times with probability distribution $P(\tau) d \tau$. The possible origins of the distribution of relaxation times in tunneling current can be the multiple other spins in the vicinity and in the tip that produce spin relaxation with different times. To obtain $1 / f$ noise in the current correlator one has to assume that $P(t) \sim 1 / \tau$ in some window of relaxation times, as was mentioned earlier.

The power spectrum of the electric current, using Eq.(10) is:

$$
\begin{gathered}
\frac{\left\langle I_{\omega}^{2}\right\rangle}{I^{2}}=\left(\sinh \left[\frac{J S}{2 F_{0}}\right]\right)^{2} \sum_{i=x, y, z} \int \frac{d \omega_{1}}{2 \pi} \frac{1}{\left|\omega-\omega_{1}\right|}\left\langle\left(S^{i}\right)_{\omega_{1}}^{2}\right\rangle \simeq \\
\left(\sinh \left[\frac{J S}{2 F_{0}}\right]\right)^{2} \frac{1}{\left[\left(\omega-\omega_{L}\right)^{2}+\gamma^{2}\right]^{1 / 2}}
\end{gathered}
$$

Where we used the low frequency asymptotics for spin current Eq. 10) and assumed Lorenzian for spin correlation function. We get finally :

$$
\frac{\left\langle I_{\omega}^{2}\right\rangle}{I^{2}} \simeq a / \pi \frac{1}{\left((\delta \omega)^{2}+\gamma\right)^{1 / 2}}
$$


Where we introduced the detuning parameter $\delta \omega=\omega-\omega_{L}$.

The signal to noise ratio is controlled by the parameter $a$ and is:

$$
\frac{\left\langle I_{\omega}^{2}\right\rangle}{I^{2}} \simeq \frac{a}{\gamma \pi}
$$

Without the microscopic model it is impossible to know exactly what is the magnitude of $a, N, \epsilon$. Self consistent treatment would give the scattering rate $\gamma \sim 10^{6} \mathrm{~Hz}$ that will be determined by the maximum of the intrinsic decay due to backaction or the extrinsic decay due to environment. As a guidance we take available STM data for the tunneling current noise [8, 9, 10, 11]. In these data the $1 / f$ noise is clearly seen above the high frequency noise floor below 100kHz. From Hodge formula we estimate $a \sim 10^{-2}$. We take $N \sim 10$ similar to mass current case, as estimated in [1]. It is convenient to relate the power spectrum of the current fluctuation at the peak at $\omega=\omega_{L}$ to the shot noise power spectrum $\left\langle I_{\text {shot }}^{2}(\omega)\right\rangle=e I$. S/N ratio is in this case is bound from above by the number on the order of unity

$$
\frac{\left\langle I_{\omega}^{2}\right\rangle}{\left\langle I_{\text {shot }}^{2}(\omega)\right\rangle} \simeq \frac{a}{N \pi \gamma \tau_{e}} \sim O(1)
$$

where we wrote explicitly the dependence of the current fluctuation on $N$.

\section{RANDOM SAMPLING}

In the previous section we discussed the conditions in which the random orientation of the spins of the tunneling electrons can, through interaction with a single precessing spin, give a signal at the Larmor frequency. Nevertheless, this is only part of the picture. Since the tunneling electrons are probing a periodic precessional motion adiabatically, sequential tunneling and temporal correlations are essential, as will be discussed below, for getting a signal with a narrow linewidth.

One of the most important characteristic of the signals detected with ESR-STM is their linewidth. the linewidth might reflect both the lifetime of the spin state, and the back action effect of the tunneling electrons on the precessing spin. However, it is necessary to discuss an additional source of the linewidth which is directly related to the way in which we probe the precession. We claim here that the periodic precession of the spin is sampled by the tunneling electrons. For each electron the precessing spin looks static (Adiabatic process). The simplest case which will be discussed here, is that the tunneling times of the tunneling electrons are uncorrelated. (As will be shown this is not a realistic assumption). In this case we can say that the sampling times obey an exponential distribution (Poisson process). We show here using the $1 / \mathrm{f}$ spin noise model described above that this is in accordance with the asymmetric lineshapes observed in the experiments. Also such a sampling process, should lead to a rapid increase in the linewidth when the magnetic field is increased. The data published so far, show such a trend.

We recall that in a tunneling current of $1 \mathrm{nA}$ there are $6.25 \times 10^{9}$ electrons/sec. The measurements were performed in a frequency of $2-8 \times 10^{8}$. This implies a ratio $\left(R_{t}\right)$ of $0.033-0.13$ of the average time between two tunneling electrons and the precession time. According to the Nyquist sampling theorem it is impossible to perform proper sampling of a periodic function if the sampling dwell time is above half of the time that it takes to complete one period. This is a fundamental limitation because it makes no sense to increase the field such that the precession frequency rises above $3 \times 10^{9} \mathrm{~Hz}$ (for a current of one nanoampere). However it is anticipated that this should be important also in much smaller frequencies. Due to the uncorrelated nature of the tunneling current, it is expected that for a periodic function that is sampled in random, (according to the Poisson distribution) increasing the frequency means that more and more sampling times (between two consecutive tunneling events) will be larger than half of the period. This implies that at larger fields (or smaller currents) we expect that the linewidth will increase. Moreover even in one peak we anticipate that the high frequency side will be broader than the low frequency side, which implies an asymmetric lineshape that has a larger slope on the low frequency side. This is precisely the behavior that we see in the measurements.

Figs. 2,3 shows two spectra that were published already. Fig. 2 shows a frequency modulated signal that is taken from Ref. 24]. The splitting in this signal was used to prove that the frequency depends on real time on the value of the magnetic field. However it is easy to see that the original spectrum is asymmetric and distorted. A similar distortion is seen in the (already published) spectrum (Fig. 3). This spectrum was taken from Ref. [26]. The shape of the spectrum is completely asymmetric and looks very similar to the Poisson distribution function. As discussed 


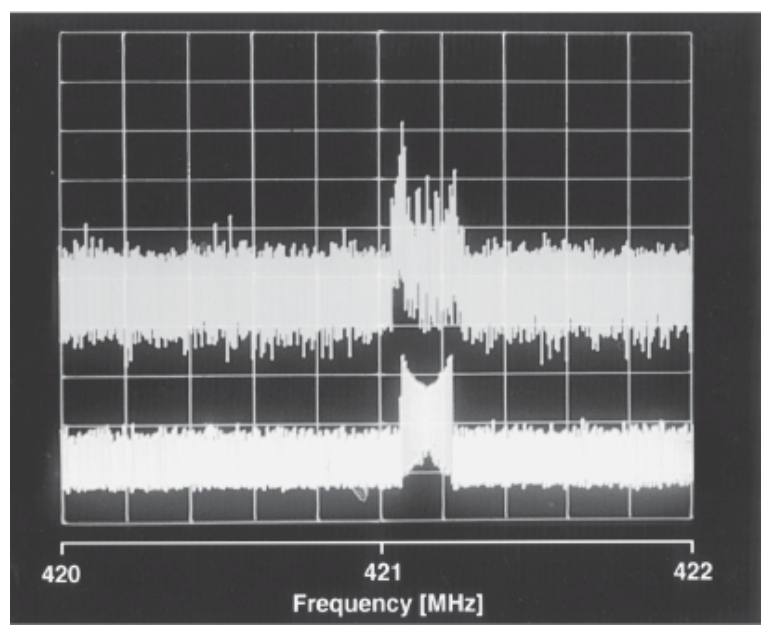

FIG. 2: A frequency modulated signal in a field of $150 \mathrm{G}$. The field modulation parameters are $\Delta \mathrm{H}=27 \mathrm{mG}$ and the modulation frequency is $300 \mathrm{KHz}$. Two spectra are presented. The upper figure represents the data from the STM, lower is simulation. It is clear that the original line-shape is not symmetric. Taken from [24]

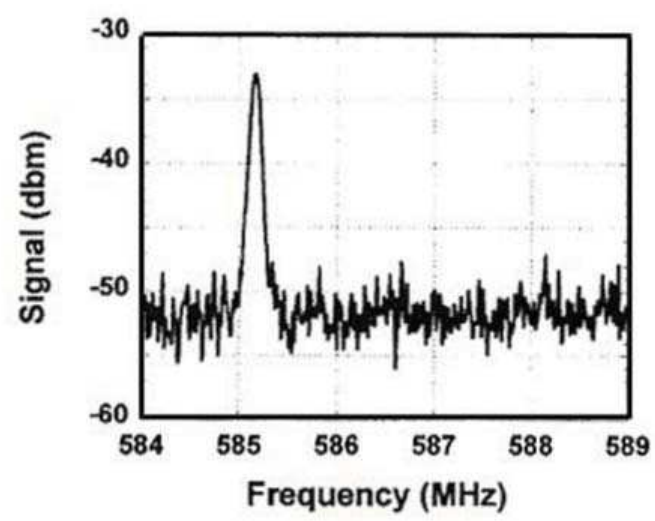

FIG. 3: An asymmetric ESR-STM spectrum of a BDPA cluster in a magnetic field of 210 G. Taken from [26]

in details in Ref. 25], this asymmetric lineshape is the cause of getting an absorption lineshape when phase sensitive detection is applied. The asymmetry was explained there as a rapid passage phenomenon. As will be shown here, we believe now that it can be explained by the physical nature of the measurement. It is important to emphasize that this is not always the case. Namely other lineshapes were also observed due to spectral diffusion. However, this represents the majority of cases.

In order to explain this trend we have done very simple simulation. In this simulation we have estimated the value represented by Eq. 8. We have taken a sinusoidal function for $S$ and a random function for $I_{s}$ which has a long time correlations (1/f peak). However, in this simulation this function is sampled in times that are determined by the exponential distribution. After that we estimated the power spectral density in the observed signal using the Welch method of spectral estimation [38].

This was done in a current of $1 \mathrm{nA}$ and at the frequencies relevant for the experiment. Each spectrum was taken for total sampling time of 0.001 seconds. By changing the frequency we changed the average number of electrons for each period. For each such spectrum the power spectrum was calculated. Fig. 4 shows the result of such a simulation for cases relevant to the experimental situation. It is clear that the calculated linewidths are much larger than anticipated from lifetime considerations. The asymmetric lineshapes observed are very similar to those observed from the experiment (compare with Fig. 3). A rapid increase in the linewidth is predicted (Fig. 5). The data we have so far support this prediction but more experiments are required (Fig.6). Using longer lifetimes gives narrower lineshapes but the overall behavior is the same. We want to emphasize that we do not take into account the backaction effect of the tunneling electrons on the precessing spin. A similar behavior is expected when the tunneling current is reduced. However this may affect other things, and we think that the dependence of the linewidth on the field is a 


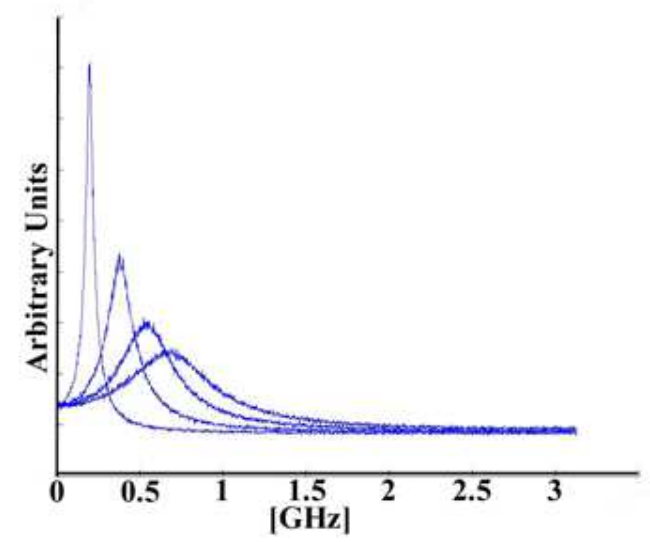

FIG. 4: The simulated lineshapes for a periodic function that is accumulated with a Poisson distribution of sampling times. A current of $1 \mathrm{nA}$ was assumed. The lineshape is clearly asymmetric.

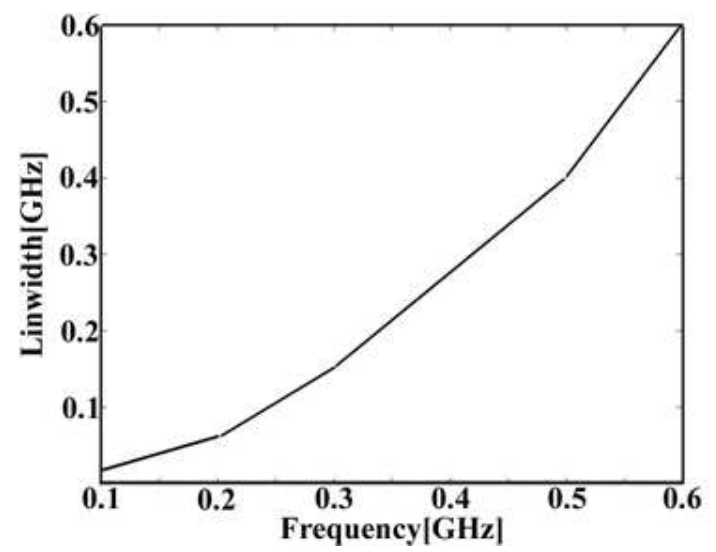

FIG. 5: The predicted linewidths as a function of the magnetic field.

more informative measurement.

The lineshape and linewidth which are shown in Figs. 4 and 5 were calculated with the $1 / \mathrm{f}$ spin noise model, but the same results will be observed from other models as well. Sampling of a sinusoidal function at times given by an exponential distribution will give similar results.

It is clear that the calculated linewidths are at least one order of magnitude larger than the experimental linewidths. This difference reveals that the exponential distribution of sampling times is totally unrealistic. Because of the small size of the tunneling region in the STM tip, two electrons can not tunnel at the same time because of the strong electrostatic repulsion between them. Thus the exponential distribution has to be modified such that some temporal correlations will be introduced in the tunneling times. We are currently trying to get more data on the linewidths in different magnetic fields, currents and bias voltages, with the hope that ESR-STM lineshapes and linewidths will reveal details on the temporal correlations in the tunneling process.

\section{Conclusion}

We have proposed a 1/f noise as a mechanism of the coupling of tunneling current from the STM tip to localized spin S. This mechanism allows one to detect a signal from single spin even in the case when there is no dc spin polarization of the tunneling current. Instead we argue that the electric current will have a contribution coming from the coupling of spin fluctuating current to the local spin. The best way to detect a single spin in this approach is to perform a difference experiment where the noise spectum is taken at the local spin site and then at the non-magnetic site elsewhere on the surface. The difference of two noise spectra would reveal the localized spin contribution. We find the signal to noise ratio for this model to be on the order $\mathrm{S} / \mathrm{N} \sim 1$. We discussed the affect of random sampling times on the linewidths. The results explain the assymmetric lineshapes commonly observed with ESR-STM. The results predict a rapid increase of the linewidth with the field. The linewidths calculated are too broad which indicates 


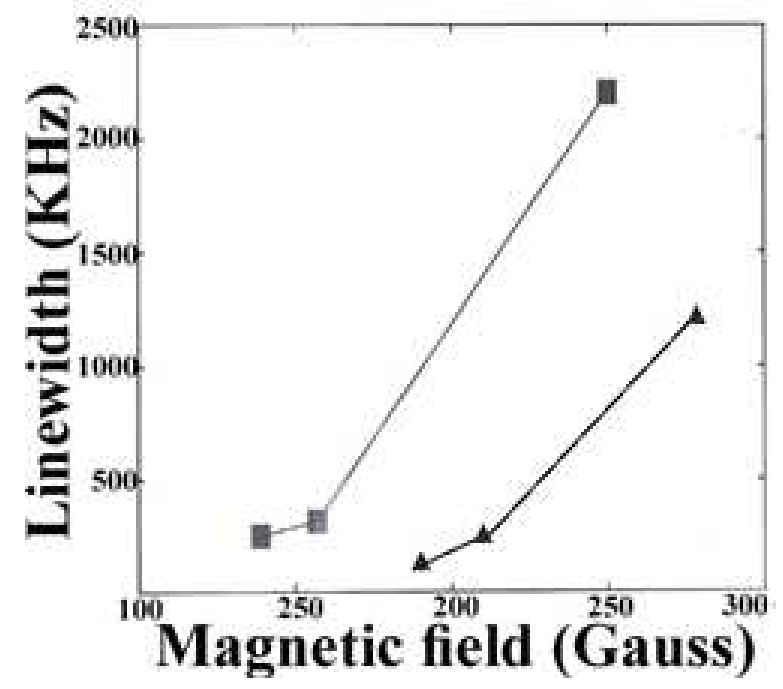

FIG. 6: The linewidths as a function of the magnetic field. The upper curve is the linewidths measured for Si $P_{b}$ center 23 while the lower curve for BDPA molecules [39]

that there must be correlations between the times of the tunneling events due to Coulomb repulsion. We think that linewidth measurements can provide a lot of information on the temporal nature of the tunneling process.

Acknowledgement

We are grateful to Z. Nussinov, J.X. Zhu, A. Shnirman for useful discussions. This work was supported by a grant from the German Israel Binational Science Foundation and LDRD at Los Alamos.

References

[1] J. B. Johnson, Phys. Rev. 26, 71 (1925).

[2] R. F. Voss and J. Clarke, Nature 258317 (1975).

[3] B. A. Taft et.al. Deep Sea Research 21403 (1974).

[4] C. Wunsch, Rev. Geophys. and Space Phys. 10 1, (1972).

[5] W. Schottky, Phys. Rev. 28 74, (1926).

[6] P. Dutta, P. Dimon and P. M. Horn, Phys. Rev. Lett. 43646 (1979).

[7] P. Dutta and P. M. Horn, Rev. Mod. Phys. 53, 497 (1981).

[8] R. Möller, A. Esslinger and B. Koslowski, Appl. Phys. Lett. 55, 2360 (1989).

[9] R. Möller, A. Esslinger, and B. Koslowski, J. Vac. Sci. Technol. A 8, 590 (1990).

[10] R. Möller, C. Baur, A. Esslinger and P. K?rz, J. Vac. Sci. Technol. B 9, 609 (1991).

[11] K. Maeda, S. Sugita, H. Kurita, M. Uota, S. Uchida, M. Hinomuro and Y. Mera, J. Vac. Sci. Technol. B 12, 2140 (1994).

[12] F. N. Hooge, Phys. Lett. A 29, 139 (1969).

[13] M. Ocio, M. Bouchiat and P. Monod, J. Magn. Magn. Mater. 54-57, 11 (1986).

[14] R. H. Koch, W. Reim, A. P. Malozemoff and M. B. Ketchen, J. Appl. Phys. 61, 3678 (1987).

[15] M. B. Weissman and N. E. Israeloff, J. Appl. Phys. 67, 4884 (1990).

[16] S. I. Woods, J. R. Kirtley, S. Sun and R. H. Koch, Phys. Rev. Lett. 87137205 (2001).

[17] V. Podzorov, M. Vehara, M. E. Gershenson, T. Y. Koo and S. -W. Cheong, Phys. Rev. B 61 R3784 (2000).

[18] B. Raquet, M. Viret, M. Coster, M. Baibich, M. Pannetier, M. Blanco-Mantecon, H. Rakoto, A. Maignan, S. Lambert and C. Fermon, J. Magn. Magn. Mater. 258-259, 119 (2003).

[19] T. W. Griswold, A. F. Kip and C. Kittel, Phys. Rev. 88951 (1952).

[20] G. Feher and A. Kip, Phys. Rev. 98, 337 (1955).

[21] F. J. Dyson, Phys. Rev. 98, 349 (1955).

[22] Y. Manassen, R. J. Hamers, J. E. Demuth and A. J. Castellano Jr. Phys. Rev. Lett. 62, 2531 (1989).

[23] Y. Manassen, E. Ter-Ovanesyan, D. Shachal and S. Richter, Phys. Rev. B 48, 4887 (1993).

[24] Y. Manassen, J. Magn. Reson. 126, 133 (1997).

[25] Y. Manassen, I. Mukhopadhyay and N. Ramesh Rao, Phys. Rev. B 61, 16223 (2000).

[26] C. Durkan and M. E. Welland, Appl. Phys. Lett. 80, 458 (2002).

[27] H. C. Manoharan, Nature, 416, 24 (2002). 
[28] G. P. Berman, G. W. Brown, M. E. Hawley and V. I. Tsifernovich, Phys. Rev. Lett. 87, 097902-1 (2001).

[29] D. Mozyrsky, F. Fedichkin, S. A. Gurvitz and G. P. Berman, Phys. Rev. B, 66, 161313 (2002).

[30] A. V. Balatsky, Y. Manassen and R. Salem, Phil. Mag. B 82, 1291 (2002).

[31] A. V. Balatsky, Y. Manassen and R. Salem, Phys. Rev. B 66, 195416 (2002).

[32] J. X. Zhu and A. V. Balatsky, Phys. Rev. Lett. 89, 286802 (2002).

[33] A. V. Balatsky and I. Martin, cond-mat/01122407

[34] L. S. Levitov and E. I. Rashba, Phys. Rev. B67, 115324 (2003)

[35] Z. Nussinov, et.al, Phys. Rev. B68, 085402, (2003)

[36] L.Bulaevskii, M Hruska and G. Ortiz, Phys.Rev.B68, 125415, (2003).

[37] A. Shnirman, D. Mozyrsky and I. Martin, cond-mat/0211618

[38] P.D. Welch, "The use of fast Fourier Transform for the Estimation of Power Spectra: A Method Based on Time Averaging over Short Modified Periodgrams", IEEE Trans. Audio. Eletroacoust" AU-15, 70 (1967).

[39] C. Durkan Contemp. Phys. 45, 1, (2004). 